\title{
Investigação do equilíbrio de adsorção de diferentes ácidos orgânicos em carvão ativado comercial
}

Massariol, P. S. ${ }^{1 *}$; Bazzarella, A. Z. ${ }^{1}$; Coura, J. C. ${ }^{1}$; Duarte, E. B. ${ }^{2}$; Ferraz, B. R. L. ${ }^{3}$; Maradini, G. S. ; Mota, M. V. de C. ${ }^{1}$; Oliveira, L. G. ${ }^{2}$; Polastreli, D. L. ${ }^{2}$; Santana, D. F. ; Santos, C. P. ${ }^{2}$; Nascimento, W.A.R. ${ }^{2}$; Soares, C. O. ${ }^{2}$; Profeti, L. P. R. ${ }^{1,2,4}$; Profeti, D. ${ }^{1,2,4}$

${ }^{1}$ Programa de Pós-Graduação em Engenharia Química, Universidade Federal do Espírito Santo, Alegre, ES, Brasil.

${ }^{2}$ Programa de Pós-Graduação em Agroquímica, Universidade Federal do Espírito Santo, Alegre, ES, Brasil.

${ }^{3}$ Departamento de Biologia, Universidade Federal do Espírito Santo, Alegre, ES, Brasil.

${ }^{4}$ Departamento de Química e Física, Universidade Federal do Espírito Santo, Alegre, ES, Brasil.

* e-mail: polyanamassariol@hotmail.com

\section{Resumo}

A adsorção de compostos orgânicos em carvão ativado comercial (CAC) é uma técnica largamente empregada no tratamento de efluentes industriais. Tal material apresenta diversas características que 0 tornam um eficiente adsorvente, entre elas alta área específica e diferentes grupos funcionais. Devido à importância do conhecimento de técnicas de remoção de substâncias poluentes, estudos envolvendo principalmente a adsorção de ácido acético em carvão ativo são amplamente difundidos e utilizados como práticas laboratoriais no meio acadêmico. Dentro deste contexto, a obtenção da isoterma de adsorção consiste em uma proposta para o ensino-aprendizagem do fenômeno adsortivo em cursos de graduação e pós-graduação que abordam o tema de preservação ambiental. Assim, o objetivo deste trabalho foi avaliar o equilíbrio de adsorção de diferentes ácidos orgânicos (metanoico, etanoico e propanoico) em CAC, utilizando os modelos das isotermas de Langmuir e Freundlich. As concentrações iniciais e finais dos ácidos foram determinadas por titulação com soluções padronizadas de $\mathrm{NaOH}$. O processo de adsorção teve duração de 5 horas, conduzido à temperatura ambiente, sob agitação. Com base nos resultados obtidos, verificou-se que o equilíbrio de adsorção dos ácidos seguiu o modelo proposto por Freundlich, embora os dados tenham apresentado bom ajuste para o modelo de Langmuir. A percentagem de remoção foi de aproximadamente 79,02\%, 61,78\% e 52,72\% para o ácido propanoico, etanoico e metanoico, respectivamente. Com isso, o experimento realizado permitiu avaliar a influência do tamanho das moléculas em processos adsortivos, possibilitando assim, compreender as interações entre o CAC e os diferentes ácidos orgânicos. Além disso, o experimento mostrou-se viável para ser aplicado como instrumento de ensino, pois auxiliou no processo de construção do conhecimento dos conceitos básicos envolvendo equilíbrio de adsorção e tratamento de efluentes.

\section{Abstract}

The adsorption of organic compounds on commercial activated carbon (CAC) is a technique widely used in the treatment of industrial effluents. Such material presents several characteristics that make it an efficient adsorbent, such as high specific area and different functional groups. Due to the importance of the understanding of techniques for removal of polluting substances, studies involving mainly the adsorption of acetic acid in activated carbon are widely used as laboratory practices in the academia. In this context, the obtainment of the adsorption isotherm consists of a proposal for teaching-learning of the adsorptive 
phenomenon in undergraduate and postgraduate courses that address the theme of environmental preservation. Thus, the objective of this work was to evaluate the adsorption equilibrium of different organic acids (methanoic, ethanoic and propanoic) on CAC using the Langmuir and Freundlich isotherm models. The initial and final concentrations of the acids were determined by titration with standard $\mathrm{NaOH}$ solutions. The adsorption process lasted for 5 hours, brought to room temperature with stirring. Based on the results obtained, it was verified that the adsorption equilibrium of the acids followed the model proposed by Freundlich, although the data presented a good fit for the Langmuir model. The removal percentage was approximately $79.02 \%, 61.78 \%$ and $52.72 \%$ for propanoic, ethanoic and methanoic acid, respectively. Thus, the experiment allowed the evaluation of the influence of molecule size in adsorptive processes, allowing an understanding of the interactions between CAC and different organic acids. In addition, the experiment demonstrated to be feasible to be applied as a teaching tool as it supported the knowledge construction process of basic concepts involving adsorption equilibrium and treatment of effluents.

Keywords (Palavras chaves): Adsorption, Activated Charcoal, Carboxylic Acids, Isotherms.

\section{Introdução}

O fenômeno da adsorção tem sido abordado no ensino em ciências ambientais com a finalidade de estudar as propriedades físico-químicas envolvidas na interação entre diferentes adsorbatos e adsorventes, como também demonstrar a importância de técnicas de remoção de contaminantes por métodos alternativos àqueles utilizados convencionalmente. Como exemplo, a prática experimental desenvolvida por Bueno e Degrève [1], amplamente difundida no âmbito do ensino de graduação e pós-graduação, aborda os conceitos de equilíbrio de adsorção de ácido etanoico em carvão ativado. As práticas laboratoriais como proposta para o ensino-aprendizagem do fenômeno adsortivo podem ressaltar a importância do tratamento dos rejeitos industriais, os quais, em sua grande maioria, são nocivos ao meio ambiente. Dentre esses compostos, os principais são de origem orgânica, oriundos de indústrias químicas, petroquímicas, farmacêuticas, entre outras [2].

Várias técnicas podem ser utilizadas para remoção de compostos orgânicos, tais como degradação fotocatalítica, processos oxidativos avançados, membranas de nanofiltração e osmose reversa, oxidação biológica e troca iônica, porém demandam elevado gasto energético e alto custo operacional [3]. Dessa forma, a adsorção é uma alternativa mais acessível, visto que é uma técnica relativamente simples, sem gasto energético e de baixo custo, sendo o carvão ativado o material adsorvente mais empregado. A alta eficácia desse material é ocasionada em virtude de sua porosidade e presença de diferentes grupos funcionais em sua superfície [4].

Processos adsortivos de ácidos orgânicos de baixo peso molecular em carvão ativado comercial dependem das características físico-químicas do adsorvente, da dimensão da cadeia carbônica do adsorvato e das condições da solução [5]. As moléculas ou íons do adsorvato tendem a fluir do meio aquoso para a superfície do adsorvente até que a concentração de soluto na fase líquida permaneça constante, assim o sistema terá atingido o equilíbrio e a capacidade de adsorção do adsorvente será determinada. Assim, o estudo do equilíbrio de adsorção dessas moléculas é de grande importância para obtenção de informações sobre processos de separação por adsorção.

O estudo do equilíbrio de adsorção pode ser realizado por diferentes modelos de isotermas de adsorção. Os modelos mais utilizados são os de Langmuir e Freundlich, os quais fornecem parâmetros como, por exemplo, a previsão da capacidade máxima de adsorção do material e a constante de equilíbrio de adsorção [6].

O objetivo desse trabalho foi avaliar 0 equilíbrio de adsorção de diferentes ácidos orgânicos (metanoico, etanoico e propanoico) em Carvão Ativo Comercial (CAC), utilizando os modelos das isotermas de Langmuir e Freundlich e possibilitar a aplicação do 


\section{Encontro Científico de Física Aplicada}

experimento em cursos de graduação e pósgraduação, a fim de ampliar conhecimentos dos conceitos envolvendo equilíbrio de adsorção e técnicas de tratamento de efluentes.

\section{Metodologia}

A avaliação da remoção dos ácidos orgânicos pelo CAC foi realizada com ensaios em batelada. Para esses experimentos, as soluções dos ácidos orgânicos, com concentrações variando entre 0,5 e $0,01 \mathrm{~mol} \mathrm{~L}^{-1}$, foram preparadas contendo também ácido clorídrico a $1,0 \times 10^{-3} \mathrm{~mol} \mathrm{~L}^{-1}$. Esta condição minimiza a dissociação dos os ácidos orgânicos em solução. O tempo de contato, utilizado neste estudo para o sistema atingir o equilíbrio, foi de 5 horas à $25^{\circ} \mathrm{C}$ sob agitação a $100 \mathrm{rpm}$.

Após o equilíbrio, as concentrações finais dos ácidos orgânicos remanescente nas soluções foram determinadas por titulação de neutralização, em triplicata, com $\mathrm{NaOH} 0,5$ e $0,05 \mathrm{~mol} \mathrm{~L}^{-1}$, previamente padronizados. Os valores de concentrações iniciais e de equilíbrio obtidos foram utilizados para a obtenção das isotermas de adsorção, onde foram avaliados os modelos de Langmuir e Freundlich.

O material adsorvente foi carvão ativado granulado tipo $119(8 \times 16)$ da marca Alphatec.

\section{Resultados e Discussão}

\subsection{Porcentagem de Remoção}

Inicialmente, avaliou-se a ocorrência de adsorção entre os ácidos orgânicos e o material adsorvente. Conforme apresentado na Figura 1, a remoção dos ácidos ocorreu para todas as amostras em diferentes concentrações iniciais.

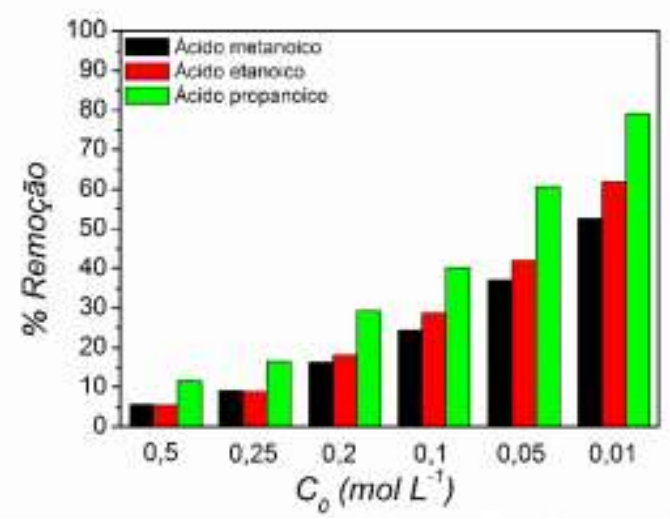

Figura 1: Porcentagem de remoção em função da concentração inicial de cada ácido.

A menor porcentagem de remoção foi de $5,46 \%$, obtida para o ácido acético na concentração de $0,5 \mathrm{~mol} \mathrm{~L}^{-1}$, enquanto que a maior porcentagem foi de $79,02 \%$ para o ácido propanoico em $0,01 \mathrm{~mol} \mathrm{~L}^{-1}$. Os resultados indicaram que o aumento das concentrações iniciais provocou a diminuição da quantidade removida de todos os ácidos analisados. Possivelmente, o aumento do valor da concentração levou à uma saturação dos sítios ativos presentes no adsorvente.

Dentre os ácidos analisados, observou-se que o ácido propanoico apresentou maior porcentagem de remoção em todas as concentrações investigadas. Isso pode ser explicado pela melhor distribuição de cargas na estrutura molecular deste ácido de maior cadeia carbônica, causando uma redução do efeito de repulsão entre as moléculas de mesma natureza. Este efeito de distribuição de cargas ao longo da molécula pode tanto favorecer o processo de adsorção como também evitar que ocorra o fenômeno de dessorção da molécula [7].

Nas concentrações iniciais variando entre 0,01 a $0,2 \mathrm{~mol} \mathrm{~L}^{-1}$ observou-se que as porcentagens de remoção aumentaram conforme aumentou-se a cadeia carbônica do ácido, ou seja, na ordem de ácido propanoico>ácido etanoico>ácido fórmico. Por outro lado, para concentrações iniciais superiores à $0,2 \mathrm{~mol}$ $\mathrm{L}^{-1}$, o ácido metanoico apresentou porcentagem de remoção sutilmente maior que o ácido etanoico, contudo ambos foram menos removidos que ácido propanoico. 
Os modelos isotérmicos de adsorção são descritos em termos de curvas que mostram a relação entre a quantidade adsorvida por unidade de massa do adsorvente e a concentração do adsorvato no equilíbrio. Através destes modelos, é possível obter a constante de equilíbrio de adsorção e a capacidade máxima de adsorção do adsorvente [6].

Os modelos matemáticos mais utilizados foram propostos por Langmuir e Freundlich. O modelo de isoterma de Langmuir descreve a adsorção em uma superfície homogênea do adsorvente, sendo que todos os sítios de adsorção são idênticos e energeticamente equivalentes e não há interação entre moléculas adsorvidas em locais vizinhos. Para sistemas de adsorção sólido-líquido, a linearização do modelo de isoterma de Langmuir pode ser expressa por:

$$
\frac{C_{e}}{q_{e}}=\frac{1}{K_{L} q_{\operatorname{máx}}}+\frac{C_{e}}{q_{\operatorname{máx}}} .
$$

Onde $q_{e}\left(\mathrm{~mol} \mathrm{~g}^{-1}\right)$ é a quantidade do adsorvato adsorvido por unidade de massa de adsorvente, $C_{e}$ $\left(\mathrm{mol} \mathrm{L}^{-1}\right)$ é a concentração de equilíbrio do adsorvato em solução e $q_{\text {máx }}\left(\mathrm{mol} \mathrm{g}^{-1}\right)$ e $K_{L}\left(\mathrm{~L} \mathrm{~g}^{-1}\right)$ são as constantes de Langmuir correspondentes à capacidade máxima de adsorção e à constante de equilíbrio de adsorção, respectivamente. Os valores de $q_{\text {máx }}$ e $K_{L}$ são determinados a partir da inclinação e intercepção da equação linearizada da isoterma de Langmuir (gráfico $C_{e} / q_{e}$ versus $C_{e}$ ).

Contrário ao modelo de Langmuir, o modelo isotérmico de adsorção proposto por Freundlich descreve a adsorção em superfície heterogênea através de um mecanismo de adsorção em multicamadas [8]. A forma linearizada da equação de Freundlich pode ser representada como:

$$
\log q e=\log K_{F}+\frac{1}{n} \log C e
$$

Onde $q_{e}\left(\mathrm{~mol} \mathrm{~g}^{-1}\right)$ representa a quantidade do adsorvato por unidade de massa de adsorvente, $C_{e}$ (mol L ${ }^{-1}$ ) é a concentração de equilíbrio do adsorvato em solução, $K_{F}$ é a constante de Freundlich $\left(\mathrm{mol} \mathrm{g}^{-1}\right)$ relacionada à capacidade de adsorção do adsorvente. $O$ valor de $n$ reflete no grau de heterogeneidade da superfície.

O equilíbrio de adsorção entre os ácidos e adsorvente do presente trabalho foi investigado com os modelos matemáticos supracitados. As isotermas obtidas estão apresentadas na Figura 2. Essa figura mostra que ocorreu um ajuste linear adequado para os dois modelos de isotermas estudados, uma vez que os coeficientes de determinação foram próximos de 1. A partir dos coeficientes, linear e angular das retas, foram obtidos os parâmetros relacionados a cada modelo isotérmico, de acordo com as equações 1 e 2 . Estes parâmetros estão dispostos na Tabela 1.

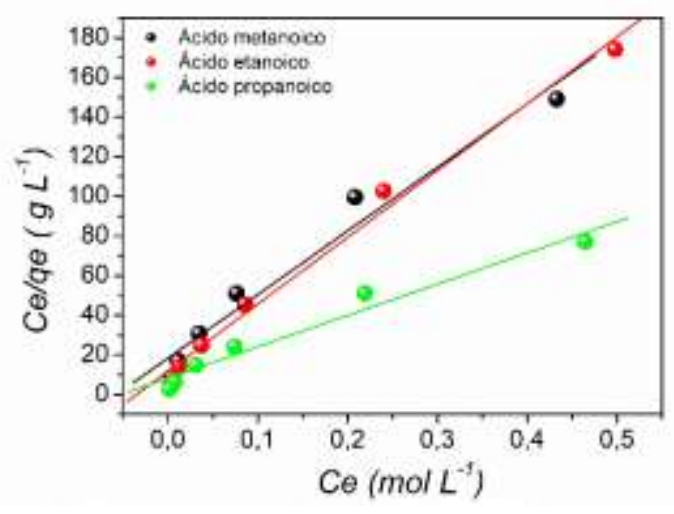

(A)

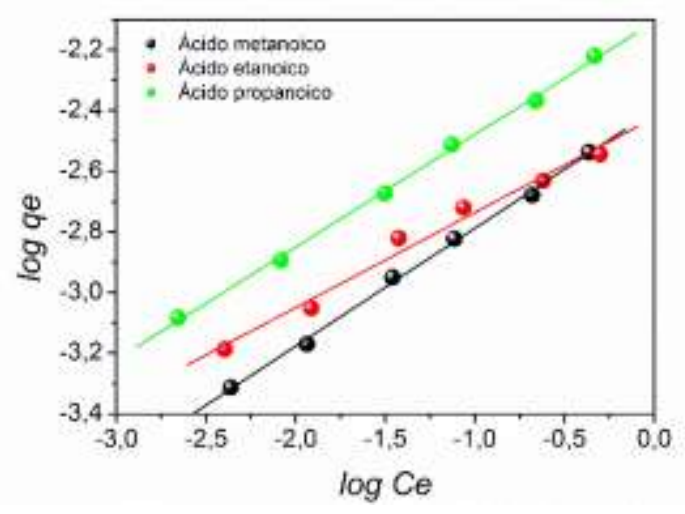

(B)

Figura 2: Isotermas de (A) Langmuir e (B) Freundlich obtidas para adsorção dos ácidos metanoico, etanoico e propanoico em CAC, T = $25 \stackrel{\circ}{\circ}$.

Os resultados obtidos indicaram que a adsorção do ácido etanoico se ajustou à isoterma de Langmuir, conforme coeficiente de determinação $\left(R^{2}\right)$ com valor de 0,995 . Portanto, podemos dizer que a 
Tabela 1: Parâmetros das Isotermas para os ácidos orgânicos

\begin{tabular}{lllllll}
\hline \multirow{2}{*}{ Ácidos orgânicos } & \multicolumn{2}{l}{ Parâmetros de Langmuir } & \multicolumn{2}{l}{ Parâmetros de Freundlich } \\
\cline { 2 - 7 } & $\begin{array}{l}\mathrm{q}_{\max } \\
\left(\mathrm{mol} \mathrm{g}^{-1}\right)\end{array}$ & $\begin{array}{l}\mathrm{K}_{\mathrm{L}} \\
\left(\mathrm{L} \mathrm{mg}^{-1}\right)\end{array}$ & $\mathrm{R}^{2}$ & $\begin{array}{l}\mathrm{K}_{\mathrm{F}} \\
\left(\mathrm{mol} \mathrm{g}^{-1}\right)\end{array}$ & $n$ & $\mathrm{R}^{2}$ \\
\hline Metanoico & $3,11 \times 10^{-3}$ & 17,55 & 0,9837 & $4,0 \times 10^{-3}$ & 2,58 & 0,9988 \\
Etanoico & $2,96 \times 10^{-3}$ & 28,51 & 0,9952 & $3,8 \times 10^{-3}$ & 3,20 & 0,9901 \\
Propanoico & $6,34 \times 10^{-3}$ & 18,91 & 0,9818 & $8,0 \times 10^{-3}$ & 2,70 & 0,9991 \\
\hline
\end{tabular}

adsorção do ácido etanoico ocorreu em sítios
homogêneos do CAC [9]. Deste modo, o modelo de isoterma de Langmuir pode ser utilizado para representar os dados experimentais de equilíbrio deste ácido. Por outro lado, a adsorção dos ácidos metanoico e propanoico se ajustou à isoterma de Freundlich, com valores de $R^{2}$ de 0,998 e 0,999, respectivamente.

Outro parâmetro importante é a capacidade máxima de adsorção dos ácidos $\left(q_{\max }\right)$ que apresenta resultado satisfatório para o ácido propanoico, seguido do ácido metanoico e etanoico.

O valor do parâmetro $n$, entre 1 e 10 , no modelo de Freundlich, indica um processo de adsorção favorável. Como mostra a Tabela 1, isto ocorreu para todos os ácidos orgânicos estudados.

\section{Conclusão}

O estudo mostrou que o carvão ativado adsorveu todos os ácidos orgânicos utilizados no experimento, sendo o ácido propanoico a espécie que apresentou maior porcentagem de remoção.

Os modelos de isotermas aplicados foram satisfatórios para descrever o equilíbrio de adsorção de todos os ácidos investigados.

O experimento mostrou-se viável para ser aplicado como instrumento de ensino, auxiliando no processo de construção do conhecimento dos conceitos básicos envolvendo equilíbrio de adsorção e tratamento de efluentes.

\section{Agradecimentos}

Os autores agradecem a CAPES e a FAPES pelo apoio financeiro.

\section{Referências}

[1] BUENO, W.A., DEGRÊVE, L. Manual de Laboratório de Físico-Química, McGraw Hill do Brasil, 1980.

[2] SCHNEIDER, E.L. Adsorção de compostos fenólicos sobre carvão ativado. Dissertação (Mestrado em Engenharia Química) - Programa de PósGraduação em Engenharia Química, Universidade Estadual do Oeste do Paraná, Toledo, 2008.

[3]FOO, K. Y.; HAMEED, B. H. Detoxification of pesticide waste via activated carbon adsorption process. Journal of Hazardous Materials, v. 175, n. 1-3, p. 1-11, 2010.

[4]LÁSZLÓ, K.; PODLOSCIELNY, P.; DABROWSKI, A. Heterogeneity of activated carbons with different surface chemistry in adsorption of phenol from aqueous solutions. Applied Surface Science, v.252 p.5752 5762, 2005.

[5] HAGHSERESHT, F.; NOURI, S.; FINNERTY, J.J.; LU, G.Q. Effects of surfaces chemistry on aromatic compound adsorption from dilute aqueous solutions by activated carbon. Journal of Physical Chemistry B, v.106 p.10935 - 10943, 2002.

[6]NASCIMENTO, R. F.; LIMA, A. C. A.; VIDAL, C. B.; MELO, D. Q.; RAULINO, G. S. C. Adsorção: aspectos teóricos e aplicações ambientais. Fortaleza, UFC, 2014. 221 p.

[7] ADEKOLA, F.A.; OBA, I.A., Biosorption of formic and acetic acids from aqueous solution using activated carbon from shea butter seed shells, Applied Water Science, v. 7, p. 2727-2736, 2017. 
[8] D.D. Do, E.A. Ustinov, H.D. Do, Porous texture characterization from gas-solid adsorption, in: E.J. Bottani, J.M.D. Tascon (Eds.), Adsorption by Carbons, Elsevier Science, 2008, pp. 239-271

[9] DOTTO, G. L.; VIEIRA, M. L. G.; GONÇALVES, J. O.PINTO, L. A. A. Remoção dos corantes azul brilhante, amarelo crepúsculo e amarelo tartrazina de soluções aquosas utilizando carvão ativado, terra ativada, terra diatomácea, quitina e quitosana: estudos de equilíbrio e termodinâmica. Quimica Nova, v. 34, n. 7, p. 1193-1199, 2011. 